\title{
Intrapartum Cardiotocography
}

National Cancer Institute

\section{Source}

National Cancer Institute. Intrapartum Cardiotocography. NCI Thesaurus. Code C92922.

The monitoring of both maternal uterine contractions and fetal heart rate during the process of labor and delivery. 